CERN-TH.6990/93

UA/NPPS-5-93

\title{
Criticality, Fractality and Intermittency in Strong Interactions
}

\author{
N.G. Antoniou, F.K. Diakonos, I.S. Mistakidis \\ Department of Physics, University of Athens, \\ GR-15771, Athens, Greece \\ and \\ C.G. Papadopoulos \\ CERN, TH Division, CH-1211, Geneva 23, Switzerland
}

\begin{abstract}
Assuming a second-order phase transition for the hadronization process, we attempt to associate intermittency patterns in high-energy hadronic collisions to fractal structures in configuration space and corresponding intermittency indices to the isothermal critical exponent at the transition temperature. In this approach, the most general multidimensional intermittency pattern, associated to a second-order phase transition of the strongly interacting system, is determined, and its relevance to present and future experiments is discussed.
\end{abstract}

CERN-TH.6990/93

August 1993 


\section{Introduction}

One of the central issues in quark-gluon plasma physics is related to the nature and structure of the quark-hadron phase transition and its measurable effects on the pattern of multihadronic states generated in a high energy-density collision of relativistic ions. Recent studies in lattice gauge theories suggest that the QCD phase transition for two flavours $(f=2)$ and massless quarks $\left(m_{u}=m_{d}=0\right)$ is of second order and this result persists even for $f=3$ provided that the mass of the strange quark is sufficiently large $\left(m_{s} \geq 100 \mathrm{MeV}\right)$ [1]. This development brings as to the hypothesis of a second-order chiral phase transition in QCD close to the real world and suggests a further exploitation of this fundamental assumption in an attempt to build-up a self-consistent dynamical scheme of strong interactions at the transition temperature $\left(T=T_{c}\right)$. In a recent work along this line F.Wilczek has argued that the hypothetical second-order chiral phase transition of the $f=2$ QCD belongs to the universality class of the $n=4$ Heisenberg magnet; therefore, in order to study the behaviour of the strongly interacting system near the critical point, one may employ the critical exponents of this magnetic system as given by the fixed point of the corresponding renormalization group [2]. In particular, the power law at $T=T_{c}$ connecting the condensate $\langle\bar{q} q\rangle$ (order parameter) with the quark mass (ordering field), $\langle\bar{q} q\rangle \sim m^{1 / \delta}$, in the limit $m \rightarrow 0\left(m=m_{u}=m_{d}\right)$ introduces a universal critical exponent $\delta$ in the strongly interacting system, which reflects the non-perturbative sector of the underlying theory. A similar power law, $\langle\rho\rangle \sim m_{\pi}^{1 / \delta}$, with the same exponent $\delta$, relating the density of hadrons (pions) in configuration space, $\langle\rho\rangle$, with the pion mass $m_{\pi}$ (chemical potential) in the chiral limit $\left(m_{\pi} \rightarrow 0\right)$, is expected to hold in the hadronic phase of a quark-gluon production process, at $T=T_{c}$. The fundamental index $\delta$ has a distinct geometrical interpretation giving the fractal dimension, $D_{F}=\frac{\delta D}{1+\delta}$, of the critical system at $T=T_{c}$, embedded in a support space of dimension $D$ [3].

In this work, adopting the hypothesis of a second-order phase transition in the strongly interacting system, we attempt to incorporate the above geometrical property of the critical system into the hadronic $S$-matrix for multiparticle production and show how the fractal structure of the critical system in configuration space may induce multidimensional intermittency patterns in momentum space, in accordance with experimental evidence. For this purpose we derive a suitable integral representation for multiparticle densities in momentum space, introducing proper light-cone variables in the treatment of the reduced $S$-matrix elements. In this approach the constraint of self-similarity (monofractality) in configuration space, required by the hypothesis of a second-order phase transition, is easily incorporated and its effect on the particle densities in momentum space is extracted. As a result, the general fractal solution leading to a complete multidimensional intermittency pattern in momentum space is obtained, expressed as a set of power laws for the factorial moments of all orders. This pattern depends on two universal indices, one of which is the critical exponent $\delta$. Therefore, a systematic comparison with experiments may give, not only an indication for the onset of a second-order phase transition in the quark-gluon system, but also a measurement of the corresponding critical exponent at $T=T_{c}$.

In section 2 the formalism leading to a complete set of hadronic densities in 
$3+1$ space-time is presented by considering the inclusive $S$-matrix treatment of a hadronic collision within the framework of inside-outside cascade dynamics [4]. In section 3, the geometrical constraint of fractality as a requirement of a second-order QCD phase transition at $T=T_{c}$ is imposed and the associated fractal solution in $3 D$ momentum space is found and discussed. In section 4 , the resulting multidimensional intermittency pattern showing strong fluctuations in small domains of momentum space, is derived. This pattern, combined with a smooth background representing conventional correlations in momentum space [5], is compared, in section 5, with the typical behaviour of the factorial moments observed in high-energy hadronic collisions. Finally, in section 6 our results are summarized and discussed and a critical comparison with other approaches is attempted.

\section{Hadronic densities in $3+1$ space-time}

Consider the inclusive process

$$
h_{A}+h_{B} \rightarrow h_{1}+h_{2}+\ldots+h_{p}+\text { anything }
$$

in the hadronization sector of the collision, defined as the component of the $S$ matrix connecting the initial hadrons $\left(h_{A}, h_{B}\right)$ with final states consisting of newly hadronized particles (at $T=T_{c}$ ) in the process of a second-order QCD phase transition. The densities $\tilde{\rho}_{c}\left(\vec{q}_{1}, \ldots, \vec{q}_{p}\right)$ in momentum space are written as follows:

$$
\left.\tilde{\rho}_{c}\left(\vec{q}_{1}, \ldots, \vec{q}_{p}\right)=\frac{E_{1} E_{2} \ldots E_{p}}{\sigma_{h}} \frac{d \sigma_{i n}^{(p)}}{d^{3} \vec{q}_{1} \ldots d^{3} \vec{q}_{p}} \sim \sum_{\left\{k_{a}\right\}} \mid\left\langle q_{1} \ldots q_{p} ;\left\{k_{a}\right\} \text { out }\right| p_{A} p_{B} \text { in }\right\rangle\left.\right|^{2}
$$

where $q_{i}=\left(\vec{q}_{i}, E_{i}\right)$ are the four-momenta of hadrons (pions) $h_{1} \ldots h_{p}$ in the final state, $\sigma_{h}$ the total cross section of the collision $h_{A}+h_{B}$ in the hadronization sector, and $d \sigma_{i n}^{(p)}$ the $p$-hadron inclusive cross section in the same sector. The summation runs over the hadronic states that build-up the missing mass in the inclusive process with a total four-momentum $k=\sum_{a} k_{a}$. The matrix elements in eq.(2.1) connect the initial state $\mid p_{A} p_{B}$; in $\rangle$ with final states $\mid$ out $\rangle$ generated at $T=T_{c}$ by the quark-hadron phase transition (the critical component of the $S$-matrix). Reducing now the $S$-matrix elements in eq.(2.1) we remove the hadrons $h_{1} \ldots h_{p}$ from the outgoing states $\mid$ out $\rangle$ and using completeness of the states $\left|\left\{k_{a}\right\}\right\rangle$ we finally obtain $[4,6]$ :

$$
\begin{aligned}
\tilde{\rho}_{c}\left(\vec{q}_{1}, \ldots, \vec{q}_{p}\right)=f_{A B} \int \prod_{i=2}^{p} d^{4} x_{1} d^{4} x_{i} d^{4} z_{i} e^{-i q_{1} x_{1}} e^{-i q_{i}\left(x_{i}-z_{i}\right)} G\left(x_{1} \ldots x_{p} ; z_{2} \ldots z_{p}\right) \\
\begin{aligned}
G\left(x_{1} \ldots x_{p} ; z_{2} \ldots z_{p}\right) & =K_{x_{1} \ldots K_{x_{p}} K_{z_{2}} \ldots K_{z_{p}}} \\
\cdot & \left\langle p_{A} p_{B}\left|T\left\{\phi\left(x_{1}\right) \ldots \phi\left(x_{p}\right)\right\} T\left\{J(0) \phi\left(z_{2}\right) \ldots \phi\left(z_{p}\right)\right\}\right| p_{A} p_{B}\right\rangle
\end{aligned}
\end{aligned}
$$

where $f_{A B}=(2 \pi)^{5} /\left(2^{p+2} W P \sigma_{h}\right)$ is a normalization factor corresponding to a total energy $W$ and incident momentum $P=\left|\vec{P}_{A}\right|=\left|\vec{P}_{B}\right|$ in the centre-of-mass system. The current $J(0)$ in eq. (2.3) is associated with the pion field $\phi(x)$ and $K_{x}$ denotes 
the corresponding Klein-Gordon operator. Changing integration variables in eq.(2.2): $x_{i}^{(+)}=x_{i}+z_{i} r_{i}=x_{i}-z_{i}(i=2, \ldots, p)$ and putting $x_{1} \equiv r_{1}$, we have:

$$
\tilde{\rho}_{c}\left(\vec{q}_{1}, \ldots, \vec{q}_{p}\right)=\frac{f_{A B}}{2^{p-1}} \int \prod_{i=2}^{p} d^{4} r_{1} d^{4} r_{i} d^{4} x_{i}^{(+)} e^{-i q_{1} r_{1}} e^{-i q_{i} r_{i}} \mathcal{G}\left(r_{1} . . r_{p} ; x_{2}^{(+)} \ldots x_{p}^{(+)}\right)
$$

Integrating over the variables $x_{i}^{(+)}$we finally obtain:

$$
\begin{aligned}
\tilde{\rho}_{c}\left(\vec{q}_{1}, \ldots, \vec{q}_{p}\right) & =\int d^{4} r_{1} \ldots d^{4} r_{p} \exp \left(-i q_{1} r_{1}-\ldots-i q_{p} r_{p}\right) \rho\left(r_{1}, . ., r_{p}\right) \\
\rho\left(r_{1}, \ldots, r_{p}\right) & =\frac{f_{A B}}{2^{p-1}} \int \prod_{i=2}^{p} d^{4} x_{i}^{(+)} \mathcal{G}\left(r_{1} \ldots r_{p} ; x_{2}^{(+)} \ldots x_{p}^{(+)}\right)
\end{aligned}
$$

In the hadronization sector the space-time development of the collision is assumed to follow inside-outside cascade dynamics; it is therefore suitable to use, in the longitudinal space-time $\left(r_{\|}, t\right)$, light-cone variables: $\tau=\left(t^{2}-r_{\|}^{2}\right)^{1 / 2}, \xi=\frac{1}{2} \ln \left|\frac{r_{\|}+t}{r_{\|}-t}\right|$. With these new coordinates we have:

$$
\begin{aligned}
q \cdot r=E t-\vec{q} \cdot \vec{r} & =\frac{1}{2}\left(E+q_{\|}\right)\left(t-r_{\|}\right)+\frac{1}{2}\left(E-q_{\|}\right)\left(t+r_{\|}\right)-\vec{q}_{\perp} \cdot \vec{r}_{\perp} \\
\exp (i q r) & =\exp \left(-i \vec{q}_{\perp} \cdot \vec{r}_{\perp}\right) \exp \left[i m_{T} \tau \cosh (\xi-y)\right]
\end{aligned}
$$

where $m_{T}$ (transverse mass) and $y$ (rapidity) are given by the well-known relations : $m_{T}=\left(q_{\perp}^{2}+m_{\pi}^{2}\right)^{1 / 2}, y=\frac{1}{2} \ln \left|\frac{E+q_{\|}}{E-q_{\|}}\right|$. The Fourier integral (2.5) is now written as follows:

$$
\tilde{\rho}_{c}\left(\vec{q}_{1}, \ldots, \vec{q}_{p}\right)=\int \prod_{i=1}^{p} \tau_{i} d \tau_{i} d \xi_{i} d^{2} \vec{r}_{\perp i} \exp \left[i \vec{q}_{\perp i} \cdot \vec{r}_{\perp i}-i \tau_{i} m_{T i} \cosh \left(y_{i}-\xi_{i}\right)\right] \rho\left(r_{1}, \ldots, r_{p}\right)
$$

where integration over the impact parameter space $\left(\vec{r}_{\perp}\right)$ has been explicitly introduced. The physical picture we have adopted in this approach (inside-outside cascade mechanism and a second-order phase transition in thermal equilibrium) requires strong constraints on the integrand $\rho\left(r_{1}, \ldots, r_{p}\right)$ in eq.(2.7). In fact since the development of the strongly interacting system in the longitudinal space-time is assumed to take place along the hyperbolas $t^{2}-r_{\|}^{2}=\tau^{2}$ and the narrow bands $\xi_{i} \approx y_{i}$ (insideoutside cascade), the support of the functions $\rho\left(r_{1}, \ldots, r_{p}\right)$ in $1+1$ space-time $(\xi, \tau)$ is restricted as follows:

$$
\rho\left(r_{1}, \ldots, r_{p}\right) \sim \delta\left(\xi_{p}-y_{p}\right) \prod_{i=1}^{p-1} \delta\left(\xi_{i}-y_{i}\right) \delta\left(\tau_{i}-\tau_{p}\right)
$$

With this constraint and after integrating over proper time variables $\tau_{i}$, eq.(2.7) is simplified as follows:

$$
\tilde{\rho}_{c}\left(y_{i}, \vec{q}_{\perp i}\right)=\int\left\{d^{2} \vec{r}_{\perp i}\right\} \exp \left(i \sum_{i=1}^{p} \vec{q}_{\perp i} \cdot \vec{r}_{\perp i}\right) \rho_{c}\left(y_{i}, \vec{r}_{\perp i} ; M_{T}\right)
$$

where $\tilde{\rho}_{c}\left(y_{i}, \vec{q}_{\perp i}\right) \equiv \tilde{\rho}_{c}\left(\vec{q}_{1}, \ldots, \vec{q}_{p}\right), \rho_{c}\left(y_{i}, \vec{r}_{\perp i} ; M_{T}\right) \equiv \rho_{c}\left(y_{1} \ldots y_{p}, \vec{r}_{\perp 1} \ldots \vec{r}_{\perp p} ; M_{T}\right),\left\{d^{2} \vec{r}_{\perp i}\right\} \equiv$ $d^{2} \vec{r}_{\perp 1} \ldots d^{2} \vec{r}_{\perp p}$ and $M_{T}=\sum_{i=1}^{p} m_{T i}$. Imposing thermal behaviour on the transverse 
mass distribution, we may write $\rho_{c}\left(y_{i}, \vec{r}_{\perp i} ; M_{T}\right)=\exp \left(-\frac{M_{T}}{T_{c}}\right) \rho_{c}\left(y_{i}, \vec{r}_{\perp i}\right)$, and since the Boltzmann factor does not affect the intermittency properties in momentum space we absorb it in $\rho_{c}\left(y_{i}, \vec{q}_{\perp i}\right)$ and finally obtain:

$$
\tilde{\rho}_{c}\left(y_{i}, \vec{q}_{\perp i}\right)=\int\left\{d^{2} \vec{r}_{\perp i}\right\} \exp \left(i \sum_{i=1}^{p} \vec{q}_{\perp i} \cdot \vec{r}_{\perp i}\right) \rho_{c}\left(y_{i}, \vec{r}_{\perp i}\right)
$$

where $\rho_{c}\left(y_{i}, \vec{r}_{\perp i}\right)$ represents hadronic densities in configuration space at $T=T_{c}$.

We consider the integral representation (2.10) as a model equation, based on rather general assumptions. It connects, at $T=T_{c}$, observable density fluctuations in $3 D$ momentum space $\left(y, \vec{q}_{\perp}\right)$ with corresponding density fluctuations in $3 D$ configuration space $\left(y, \vec{r}_{\perp}\right)$. It is, therefore, a suitable equation for studying multidimensional intermittency patterns associated to self-similar structures of the hadronic system in real space, at $T=T_{c}$.

\section{Criticality and Fractality}

Our basic hypothesis in this treatment is a simplified picture for the hadronization process, in a high energy-density collision, as a static, second-order QCD phase transition in thermal equilibrium. It generates at $T=T_{c}$ strong density fluctuations resulting in the development of a fractal dimension $D_{F}$ in real space according to the standard behaviour of thermal systems undergoing such a transition and characterized by an isothermal critical exponent $\delta$ at $T=T_{c}\left(D_{F}=\frac{\delta D}{1+\delta}\right)$ [3]. In this picture it is natural to assume that the hypothetical second-order QCD phase transition manifests itself as a critical behaviour of the classical $3 D$ system (hadronic fluid) specified at $T=T_{c}$ by the complete set of densities $\left\{\rho_{c}\left(y_{1} \ldots y_{p}, \vec{r}_{\perp 1} \ldots \vec{r}_{\perp p}\right), p=1,2, \ldots\right\}$ introduced in eq.(2.10). With this interpretation, the characteristic power law of the chiral phase transition at $T=T_{c},\langle\bar{q} q\rangle \sim m^{1 / \delta}$, valid in the quark phase $(m$ is the quark mass), must have its counterpart in the hadronic phase, with the same critical exponent $\delta$ :

$$
\langle n\rangle \sim \mu^{1 / \delta} \quad\langle n\rangle=\frac{\partial \ln Q_{c}}{\partial \mu} \quad\left(T=T_{c}\right)
$$

where $\langle n\rangle$ is the average multiplicity of hadrons, $Q_{c}$ the grand partition function at $T=T_{c}$, given in terms of the densities $\rho_{c}\left(y_{i}, \vec{r}_{\perp i}\right)$, and $\mu$ the chemical potential for the production process, normally identified with the pion mass $\left(\mu=m_{\pi}\right)$.

For completeness, one can easily verify that the critical behaviour (3.1) of a classical system in $D$ dimensions is associated to a self-similar structure in configuration space corresponding to a fractal dimension $D_{F}=\frac{\delta D}{1+\delta}$. For this purpose, consider the grand partition function $Q_{c}(\mu, V)$ of such a system, at $T=T_{c}$, in terms of the densities $\rho_{c}\left(\vec{x}_{1}, \ldots, \vec{x}_{p}\right)(p=1,2 \ldots)$ as follows:

$$
Q_{c}(\mu, V)=\sum_{p} \frac{(z-1)^{p}}{p !} \int_{V}\left\{d^{D} \vec{x}_{i}\right\} \rho_{c}\left(\vec{x}_{1}, \ldots, \vec{x}_{p}\right)
$$

where $z=\exp \left(\mu / T_{c}\right)$ and $V$ is the volume of the system in $D$ dimensions. A selfsimilar structure in configuration space with fractal dimension $D_{F}$ requires:

$$
\rho_{c}\left(\lambda \vec{x}_{1}, \ldots, \lambda \vec{x}_{p}\right)=\lambda^{\left(D_{F}-D\right) p} \rho_{c}\left(\vec{x}_{1}, \ldots, \vec{x}_{p}\right)
$$


and in the limit $\mu \rightarrow 0$ eqs.(3.2) and (3.3) lead to a scaling property, $Q_{c}(\mu, V)=$ $F\left(\mu V^{D_{F} / D}\right)$, as follows:

$$
Q_{c}(\mu, V)=\sum_{p}\left(\frac{\mu V^{D_{F} / D}}{T_{c}}\right)^{p} \frac{1}{p !} \int_{\left|\vec{\xi}_{i}\right| \leq 1}\left\{d^{D} \vec{\xi}_{i}\right\} \rho_{c}\left(\vec{\xi}_{1}, \ldots, \vec{\xi}_{p}\right)
$$

In the thermodynamic limit $(V \rightarrow \infty)$ the scaling form $Q_{c}=F\left(\mu V^{D_{F} / D}\right)$ combined with the normal boundary condition $Q_{c} \sim \exp [f(\mu) V]$, leads to the critical behaviour (3.1) with $\delta=\frac{D_{F}}{D-D_{F}}$, giving finally $D_{F}=\frac{\delta D}{1+\delta}$. This simple relation of the critical exponent $\delta$ with the fractal dimension $D_{F}$ of the system at $T=T_{c}$ together with eq. (2.10) provides a basic ingredient in our approach since it makes the connection of the hadronic intermittency with a second-order phase transition [7] more transparent and easier to understand.

In this context and in order to establish a link between intermittency patterns in momentum space with criticality of the hadronic system at $T=T_{c}$ one has to search, using eq.(2.10), for the most general class of fractals in momentum space $\left(y, \vec{q}_{\perp}\right)$ generated by a self-similar structure in $3 D$ configuration space $\left(y, \vec{r}_{\perp}\right)$ corresponding to a fractal dimension $D_{3}=\frac{3 \delta}{1+\delta}$. For this purpose and in order to impose selfsimilarity, we introduce new coordinates in both spaces, appropriate to the fact that the system, being finite, is not necessarily translational invariant. We have:

$$
\begin{array}{rll}
\vec{r}_{\perp i}{ }^{\prime}=\vec{r}_{\perp i}-\vec{R}_{\perp} & (i=1, \ldots p-1) & \vec{R}_{\perp}=\frac{1}{p}\left(\vec{r}_{\perp 1}+\ldots+\vec{r}_{\perp p}\right) \\
\vec{q}_{\perp i}{ }^{\prime}=\vec{q}_{\perp i}-\vec{Q}_{\perp i} & (i=1, \ldots p-1) & \vec{Q}_{\perp}=\frac{1}{p}\left(\vec{q}_{\perp 1}+\ldots+\vec{q}_{\perp p}\right) \\
y_{i}^{\prime}=y_{i}-Y & (i=1, \ldots p-1) & Y=\frac{1}{p}\left(y_{1}+\ldots+y_{p}\right)
\end{array}
$$

With these new variables, the problem of finding fractals in momentum space, associated to self-similar structures in configuration space is formulated as follows :

$$
\begin{gathered}
\tilde{\rho}_{c}\left(y_{i}^{\prime}, \vec{q}_{\perp i}{ }^{\prime} ; Y, \vec{Q}_{\perp}\right)=\int d^{2} \vec{R}_{\perp} e^{i \vec{Q}_{\perp} \cdot \vec{R}_{\perp} \int\left\{d^{2} \vec{r}_{\perp i}{ }^{\prime}\right\}} \\
\cdot \quad \exp \left(i \sum_{\mu, \nu}^{p-1} \alpha_{\mu \nu} \vec{q}_{\perp \mu}{ }^{\prime} \vec{r}_{\perp \nu}{ }^{\prime}\right) \rho_{c}\left(y_{i}^{\prime}, \vec{r}_{\perp i}{ }^{\prime} ; Y, \vec{R}_{\perp}\right) \\
\rho_{c}\left(\lambda y_{i}^{\prime}, \lambda \vec{r}_{\perp i}{ }^{\prime} ; Y, \vec{R}_{\perp}\right)=\lambda^{\left(D_{3}-3\right)(p-1)} \rho_{c}\left(y_{i}^{\prime}, \vec{r}_{\perp i}{ }^{\prime} ; Y, \vec{R}_{\perp}\right) \\
\tilde{\rho}_{c}\left(\lambda y_{i}^{\prime}, \lambda \vec{q}_{\perp i}{ }^{\prime} ; Y, \vec{Q}_{\perp}\right)=\lambda^{\left(\tilde{D}_{3}-3\right)(p-1)} \tilde{\rho}_{c}\left(y_{i}^{\prime}, \vec{q}_{\perp i}{ }^{\prime} ; Y, \vec{Q}_{\perp}\right)
\end{gathered}
$$

We have used eq.(2.10) together with appropriate self-similarity conditions corresponding to fractal dimensions $D_{3}$ and $\tilde{D}_{3}$ in configuration and momentum space respectively. The coefficients $\alpha_{\mu \nu}$ in eq.(3.6) are fixed by the transformation equations (3.5). From eqs.(3.6) to (3.8) one easily obtains stronger self-similarity constraints as follows:

$$
\begin{aligned}
& \tilde{\rho}_{c}\left(\lambda^{2} y_{i}^{\prime}, \vec{q}_{\perp i}{ }^{\prime} ; Y, \vec{Q}_{\perp}\right)=\lambda^{\left(\tilde{D}_{3}+D_{3}-4\right)(p-1)} \tilde{\rho}_{c}\left(y_{i}^{\prime}, \vec{q}_{\perp i}{ }^{\prime} ; Y, \vec{Q}_{\perp}\right) \\
& \tilde{\rho}_{c}\left(y_{i}^{\prime}, \lambda^{2} \vec{q}_{\perp i}{ }^{\prime} ; Y, \vec{Q}_{\perp}\right)=\lambda^{\left(\tilde{D}_{3}-D_{3}-2\right)(p-1)} \tilde{\rho}_{c}\left(y_{i}^{\prime}, \vec{q}_{\perp i}{ }^{\prime} ; Y, \vec{Q}_{\perp}\right)
\end{aligned}
$$


or in a unified form:

$$
\tilde{\rho}_{c}\left(\lambda y_{i}^{\prime}, \mu \vec{q}_{\perp i}{ }^{\prime} ; Y, \vec{Q}_{\perp}\right)=\lambda^{\frac{1}{2}\left(\tilde{D}_{3}+D_{3}-4\right)(p-1)} \mu^{\frac{1}{2}\left(\tilde{D}_{3}-D_{3}-2\right)(p-1)} \tilde{\rho}_{c}\left(y_{i}^{\prime}, \vec{q}_{\perp i}{ }^{\prime} ; Y, \vec{Q}_{\perp}\right)
$$

The geometrical interpretation of eq.(3.11) is that the fractal $\tilde{F}_{3}$ in $3 D$ momentum space, generated by a fractal $F_{3}$ in configuration space, has necessarily the structure of a Cartesian product of two fractals, $\tilde{F}_{1}$ and $\tilde{F}_{2}$, in rapidity and transverse momentum space respectively, $\tilde{F}_{3}=\tilde{F}_{1} \times \tilde{F}_{2}[8]$. The fractal dimensions involved in this structure are: $\tilde{D}_{1}=\frac{1}{2}\left(D_{3}+\tilde{D}_{3}-2\right), \tilde{D}_{2}=\frac{1}{2}\left(\tilde{D}_{3}-D_{3}+2\right)$ satisfying the Cartesian product relation $\tilde{D}_{1}+\tilde{D}_{2}=\tilde{D}_{3}$. Similarly, in configuration space one obtains:

$$
\rho_{c}\left(\lambda y_{i}^{\prime}, \mu \vec{r}_{\perp i}{ }^{\prime} ; Y, \vec{R}_{\perp}\right)=\lambda^{\frac{1}{2}\left(\tilde{D}_{3}+D_{3}-4\right)(p-1)} \mu^{\frac{1}{2}\left(D_{3}-\tilde{D}_{3}-2\right)(p-1)} \rho_{c}\left(y_{i}^{\prime}, \vec{r}_{\perp i}{ }^{\prime} ; Y, \vec{R}_{\perp}\right)
$$

showing that the fractal $F_{3}$ has also the structure of a Cartesian product, $F_{3}=$ $F_{1} \times F_{2}$, of two fractals, one in rapidity $\left(F_{1}\right)$ and the other in impact parameter space $\left(F_{2}\right)$. The corresponding fractal dimensions in this case are: $D_{1}=\frac{1}{2}\left(D_{3}+\tilde{D}_{3}-2\right)$, $D_{2}=\frac{1}{2}\left(D_{3}-\tilde{D}_{3}+2\right)$ and $D_{3}=D_{1}+D_{2}$.

The realization of this geometry in terms of particle densities in momentum space leads to the general form:

$$
\tilde{\rho}_{c}\left(y_{i}^{\prime}, \vec{q}_{\perp i}{ }^{\prime} ; Y, \vec{Q}_{\perp}\right)=\rho_{c 1}\left(y_{i}^{\prime}\right) \tilde{\rho}_{c 2}\left(\vec{q}_{\perp i}{ }^{\prime}\right) \Phi_{i n v}^{(3)}\left(y^{\prime}, \vec{q}_{\perp i}{ }^{\prime} ; Y, \vec{Q}_{\perp}\right)
$$

where $\rho_{c 1}, \tilde{\rho}_{c 2}$ obey self-similarity and $\Phi_{i n v}^{(3)}$ is invariant, under scale transformations : $y_{i}^{\prime} \rightarrow \lambda y_{i}^{\prime}, \vec{q}_{\perp i} \rightarrow \mu \vec{q}_{\perp i}$ as follows:

$$
\begin{aligned}
\rho_{c 1}\left(\lambda y_{i}^{\prime}\right) & =\lambda^{\left(D_{1}-1\right)(p-1)} \rho_{c 1}\left(y_{i}^{\prime}\right) \\
\tilde{\rho}_{c 2}\left(\mu \vec{q}_{\perp i}{ }^{\prime}\right) & =\mu^{\left(\tilde{D}_{2}-2\right)(p-1)} \tilde{\rho}_{c 2}\left(\vec{q}_{\perp i}{ }^{\prime}\right) \\
\Phi_{\text {inv }}^{(3)}\left(\lambda y_{i}^{\prime}, \mu \vec{q}_{\perp i} ; Y, \vec{Q}_{\perp}\right) & =\Phi_{i n v}^{(3)}\left(y_{i}^{\prime}, \vec{q}_{\perp i} ; Y, \vec{Q}_{\perp}\right)
\end{aligned}
$$

In summary, we have shown that the most general fractal solution of eq.(2.10) in configuration and momentum space, associated to a second-order phase transition during the development of the collision, has the structure of a Cartesian product in both spaces. The geometrical characteristics of these fractals are summarized as follows: (a) in configuration space, $F_{3}=F_{1} \times F_{2}$ with $D_{3}=D_{1}+D_{2}$ and (b) in momentum space, $\tilde{F}_{3}=\tilde{F}_{1} \times \tilde{F}_{2}$ with $\tilde{F}_{1}=F_{1}, \tilde{D}_{1}=D_{1}, \tilde{D}_{2}=2-D_{2}, \tilde{D}_{3}=$ $2+D_{1}-D_{2}$. It is of interest to note that the whole pattern of fractals in both spaces depends on two universal indices $\left(D_{1}, D_{2}\right)$ which are constrained by the isothermal critical exponent $\delta, D_{1}+D_{2}=\frac{3 \delta}{1+\delta}$.

In momentum space, the pattern of fractals has to be completed by considering $1 D$ projections of $\tilde{F}_{2}$ within its support space (transverse momentum plane). Physically it is equivalent to search for $1 D$ intermittency patterns in azimuthal angle $(\delta \phi)$ or transverse momentum $\left(\delta q_{\perp}\right)$ domains. According to deterministic fractal geometry 88 if $\tilde{D}_{2}<1$ the projection of $\tilde{F}_{2}$ onto $1 D$ subspaces of the transverse momentum plane is also a fractal $\tilde{F}_{\perp 1}$ with the same fractal dimension $\tilde{D}_{\perp 1}=\tilde{D}_{2}$. Otherwise, if $\tilde{D}_{2}>1$, the $1 D$ projections of $\tilde{F}_{2}$ do not develop any fractal structure $\left(\tilde{D}_{\perp 1}=1\right)$. This property remains valid for random fractals as well and can be verified for $\tilde{F}_{2}$ as 
follows:

Consider the projection of $\tilde{F}_{2}$ onto any $1 D u$-space by integrating the densities $\tilde{\rho}_{c 2}\left(\vec{q}_{\perp i}{ }^{\prime}\right)$ over the orthogonal $v$-space $(u, v$ are usual Cartesian coordinates in $2 D$ transverse momentum). The projected densities are given by the integral:

$$
\tilde{\rho}_{\perp c}^{(1)}\left(u_{i}\right)=\int \tilde{\rho}_{c 2}\left(u_{i}, v_{i}\right) d v_{1} \ldots d v_{p-1}
$$

which, after imposing the self-similarity constraint (3.14) on $\tilde{\rho}_{c 2}$, gives:

$$
\tilde{\rho}_{\perp c}^{(1)}\left(\lambda u_{i}\right)=\lambda^{\left(\tilde{D}_{2}-1\right)(p-1)} \tilde{\rho}_{\perp c}^{(1)}\left(u_{i}\right)
$$

For $\tilde{D}_{2}<1$ eq.(3.16) represents a self-similar structure in $1 D u$-space with fractal dimension $\tilde{D}_{\perp 1}=\tilde{D}_{2}$ whereas if $\tilde{D}_{2}>1$ the singularity for $\lambda \rightarrow 0$ disappears and the projected particle densities have a smooth non-fractal behaviour. We therefore conclude that there are, in general, two possible classes of fractal patterns in momentum space, depending on the actual value of the fractal dimension $\tilde{D}_{2}$, which can be attributed to a critical behaviour of the hadronic system at $T=T_{c}$. Although $\tilde{D}_{2}>1$ cannot be excluded on geometrical grounds alone, it can be easily seen that it requires rather unrealistic values for the critical exponent $\delta$ and, therefore, can be safely ignored on physical grounds. In fact, from the general relations $\tilde{D}_{2}=2-D_{3}+D_{1}$ and $D_{3}=\frac{3 \delta}{1+\delta}\left(0<D_{1}<1\right)$, one obtains the bound $\tilde{D}_{2} \leq \frac{3}{1+\delta}$, which in the sector $\tilde{D}_{2}>1$ implies $\delta<2$. Since it is very unlikely for the critical exponent $\delta$ to get such small values in any realistic $3 D$ system undergoing a second-order phase transition [9] one may consider solution with $\tilde{D}_{2}<1$ as the only acceptable class of fractals in momentum space associated to a second-order quark-hadron phase transition. The geometrical characteristics (embedding dimensions, Cartesian products, fractal dimensions $D_{F}$ ) of this solution are summarized as follows:

$$
\begin{aligned}
& 3 D: \tilde{F}_{1}(y) \times \tilde{F}_{2}\left(q_{\perp}, \phi\right) \\
& D_{F}=2+D_{1}-D_{2} \\
& 2 D: \tilde{F}_{2}\left(q_{\perp}, \phi\right) \\
& D_{F}=2-D_{2} \\
& 2 D: \tilde{F}_{\perp 1}\left(q_{\perp}\right) \times \tilde{F}_{1}(y), \tilde{F}_{\perp 1}(\phi) \times \tilde{F}_{1}(y) \\
& D_{F}=2+D_{1}-D_{2} \\
& 1 D: \tilde{F}_{\perp 1}\left(q_{\perp}\right), \tilde{F}_{\perp 1}(\phi) \\
& D_{F}=2-D_{2} \\
& 1 D: \tilde{F}_{1}(y) \\
& D_{F}=D_{1}
\end{aligned}
$$

The physical content of the geometrical pattern (3.17) may be revealed by studying the behaviour of factorial moments in small domains of momentum space, using appropriate self-similarity relations implied by the above fractal structures. A set of power laws, dependent on two universal parameters $\left(D_{1}, D_{2}\right)$, is expected to be generated, resulting in a multidimensional intermittency pattern with a linear spectrum of indices. The details of this treatment are given in section 4 .

\section{Multidimensional Intermittency}

The scaled factorial moments, $F_{p}(\delta \Omega)$, in small domains of momentum space [10], are suitable for probing geometrical structures of form (3.17) and therefore connecting a 
second-order phase transition at the level of quark-gluon dynamics with observable multiplicity fluctuations at the level of the hadronic $S$-matrix. The basic ingredients in this approach are the integrals:

$$
F_{p}^{(3)}\left(\delta \Omega_{3}\right) \sim\left(\delta \Omega_{3}\right)^{-p} \int_{\delta \Omega_{3}} \tilde{\rho}_{c}\left(y_{i}^{\prime}, \vec{q}_{\perp i}{ }^{\prime} ; Y, \vec{Q}_{\perp}\right)\left\{d y_{i}^{\prime}\right\}\left\{d^{2} \vec{q}_{\perp i}{ }^{\prime}\right\} d Y d^{2} \vec{Q}_{\perp}
$$

$(p=2,3 \ldots)$ where integration is restricted in the vicinity of a fixed configuration: $y_{i}^{\prime}=0, \vec{q}_{\perp i}{ }^{\prime}=0, Y=Y_{0} \quad \vec{Q}_{\perp}=\vec{Q}_{\perp 0} \quad(i=1,2 \ldots, p-1)$. In this domain eq.(4.1) is simplified as follows:

$$
F_{p}^{(3)}\left(\delta \Omega_{3}\right) \sim\left(\delta \Omega_{3}\right)^{1-p} \int_{\delta \Omega_{3}} \tilde{\rho}_{c}\left(y_{i}^{\prime}, \vec{q}_{\perp i}{ }^{\prime} ; Y_{0}, \vec{Q}_{\perp 0}\right)\left\{d y_{i}^{\prime}\right\}\left\{d^{2} \vec{q}_{\perp i}{ }^{\prime}\right\}
$$

The moments $F_{p}^{(3)}\left(\delta \Omega_{3}\right)$ refer to $3 D$ intermittency but similar quantities $F_{p}^{(1)}$ and $F_{p}^{(2)}$ in one or two dimensions are easily obtained by projecting out onto appropriate momentum subspaces. Using the multiplicative form (3.13) for the densities $\tilde{\rho}_{c}\left(y_{i}^{\prime}, \vec{q}_{\perp i}{ }^{\prime} ; Y_{0}, \vec{Q}_{\perp 0}\right)$ we may write a multidimensional pattern of moments as follows :

$$
\begin{gathered}
F_{p}^{(1)}\left(\delta \Omega_{1}\right) \sim\left(\delta \Omega_{1}\right)^{1-p} \int_{\delta \Omega_{1}} \rho_{c 1}\left(y_{i}^{\prime}\right) \Phi_{i n v}^{(1)}\left(y_{i}^{\prime} ; Y_{0}\right)\left\{d y_{i}^{\prime}\right\} \\
F_{p}^{(1)}\left(\delta \Omega_{1}^{\prime}\right) \sim\left(\delta \Omega_{1}^{\prime}\right)^{1-p} \int_{\delta \Omega_{1}^{\prime}} \tilde{\rho}_{\perp c}^{(1)}\left(u_{i}\right) \Phi_{i n v}^{(1)}\left(u_{i} ; Q_{u}^{(0)}\right)\left\{d u_{i}\right\} \\
F_{p}^{(2)}\left(\delta \Omega_{2}^{\prime}\right) \sim\left(\delta \Omega_{2}^{\prime}\right)^{1-p} \int_{\delta \Omega_{2}^{\prime}} \rho_{c 1}\left(y_{i}^{\prime}\right) \tilde{\rho}_{\perp c}^{(1)}\left(u_{i}\right) \Phi_{i n v}^{(2)}\left(y_{i}^{\prime}, u_{i} ; Y_{0}, Q_{u}^{(0)}\right)\left\{d y_{i}^{\prime}\right\}\left\{d u_{i}\right\} \\
F_{p}^{(2)}\left(\delta \Omega_{2}\right) \sim\left(\delta \Omega_{2}\right)^{1-p} \int_{\delta \Omega_{2}} \tilde{\rho}_{c 2}\left(\vec{q}_{\perp i}{ }^{\prime}\right) \Phi_{i n v}^{(2)}\left(\vec{q}_{\perp i}{ }^{\prime} ; \vec{Q}_{\perp 0}\right)\left\{d^{2} \vec{q}_{\perp i}{ }^{\prime}\right\} \\
F_{p}^{(3)}\left(\delta \Omega_{3}\right) \sim\left(\delta \Omega_{3}\right)^{1-p} \int_{\delta \Omega_{3}} \rho_{c 1}\left(y_{i}^{\prime}\right) \tilde{\rho}_{c 2}\left(\vec{q}_{\perp i}{ }^{\prime}\right) \Phi_{i n v}^{(3)}\left(y_{i}^{\prime}, \vec{q}_{\perp i}{ }^{\prime} ; Y_{0}, \vec{Q}_{\perp 0}\right)\left\{d y_{i}^{\prime}\right\}\left\{d^{2} \vec{q}_{\perp i}{ }^{\prime}\right\}
\end{gathered}
$$

where $Q_{u}^{(0)}$ is the component of $\vec{Q}_{\perp 0}$ along the $u$-direction in the transverse momentum plane. The integration volumes $\delta \Omega_{D}$ are identified as follows: $\delta \Omega_{1}=\delta y, \delta \Omega_{1}^{\prime}=\delta q_{\perp}$ or $\delta \phi, \delta \Omega_{2}^{\prime}=\delta q_{\perp} \delta y$ or $\delta \phi \delta y, \delta \Omega_{2}=\delta \phi \delta q_{\perp}$ and $\delta \Omega_{3}=\delta y \delta \phi \delta q_{\perp}$. Their size is taken $\delta \Omega_{D} \sim M^{-D}$ with $M \gg 1$ and the dependence of moments on $M$ reveals the intermittency effects in the process under consideration.

The integrands in eqs.(4.3) to (4.7) obey self-similarity corresponding to codimensions $D-D_{F}=1-D_{1}, D_{2}-1, D_{2}-D_{1}, D_{2}, 1+D_{2}-D_{1}$. Therefore using the transformations $y_{i}^{\prime}=M^{-1} \theta_{i}, \vec{q}_{\perp i}{ }^{\prime}=M^{-1} \vec{s}_{i}, u_{i}=M^{-1} \gamma_{i}$, we obtain the power laws [11]:

$$
\begin{aligned}
F_{p}^{(1)}\left(\delta \Omega_{1}\right) & \sim M^{\left(1-D_{1}\right)(p-1)} \int_{\delta \omega_{1}} \rho_{c 1}\left(\theta_{i}\right) \Phi_{i n v}^{(1)}\left(\theta_{i} ; Y_{0}\right)\left\{d \theta_{i}\right\} \\
F_{p}^{(1)}\left(\delta \Omega_{1}^{\prime}\right) & \sim M^{\left(D_{2}-1\right)(p-1)} \int_{\delta \omega_{1}^{\prime}} \tilde{\rho}_{\perp c}^{(1)}\left(\gamma_{i}\right) \Phi_{i n v}^{(1)}\left(\gamma_{i} ; Q_{u}^{(0)}\right)\left\{d \gamma_{i}\right\} \\
F_{p}^{(2)}\left(\delta \Omega_{2}^{\prime}\right) & \sim M^{\left(D_{2}-D_{1}\right)(p-1)} \int_{\delta \omega_{2}^{\prime}} \rho_{c 1}\left(\theta_{i}\right) \tilde{\rho}_{\perp c}^{(1)}\left(\gamma_{i}\right) \Phi_{i n v}^{(2)}\left(\theta_{i}, \gamma_{i} ; Y_{0}, Q_{u}^{(0)}\right)\left\{d \theta_{i}\right\}\left\{d \gamma_{i}\right\} \\
F_{p}^{(2)}\left(\delta \Omega_{2}\right) & \sim M^{D_{2}(p-1)} \int_{\delta \omega_{2}} \tilde{\rho}_{c 2}\left(\vec{s}_{i}\right) \Phi_{i n v}^{(2)}\left(\vec{s}_{i} ; \vec{Q}_{\perp 0}\right)\left\{d^{2} \vec{s}_{i}\right\} \\
F_{p}^{(3)}\left(\delta \Omega_{3}\right) & \sim M^{\left(1+D_{2}-D_{1}\right)(p-1)} \\
& \cdot \int_{\delta \omega_{3}} \rho_{c 1}\left(\theta_{i}\right) \tilde{\rho}_{c 2}\left(\vec{s}_{i}\right) \Phi_{i n v}^{(3)}\left(\theta_{i}, \vec{s}_{i} ; Y_{0}, \vec{Q}_{\perp 0}\right)\left\{d \theta_{i}\right\}\left\{d^{2} \vec{s}_{i}\right\}
\end{aligned}
$$


where the integration domains have finite, $M$-independent volumes $\delta \omega_{D} \sim M^{D} \delta \Omega_{D}$. In summary, we may write the complete multidimensional intermittency pattern corresponding to fractal structures (3.17) as follows:

$$
\begin{aligned}
\ln F_{p}^{(1)}(\delta y) & =\left(1-D_{1}\right)(p-1) \ln M \\
\ln F_{p}^{(1)}(\delta \phi) & =\ln F_{p}^{(1)}\left(\delta q_{\perp}\right)=\left(D_{2}-1\right)(p-1) \ln M \\
\ln F_{p}^{(2)}\left(\delta q_{\perp}, \delta y\right) & =\ln F_{p}^{(2)}(\delta \phi, \delta y)=\left(D_{2}-D_{1}\right)(p-1) \ln M \\
\ln F_{p}^{(2)}\left(\delta \phi, \delta q_{\perp}\right) & =D_{2}(p-1) \ln M \\
\ln F_{p}^{(3)}\left(\delta y, \delta \phi, \delta q_{\perp}\right) & =\left(1+D_{2}-D_{1}\right)(p-1) \ln M
\end{aligned}
$$

with the constraint $D_{1}+D_{2}=\frac{3 \delta}{1+\delta}(\delta>2)$. A number of characteristic properties of the pattern (4.9) can be easily observed, independently of the actual values of the universal indices $D_{1}, D_{2}$ : (a) There is a certain degree of degeneracy at the level of $1 D$ and $2 D$ moments, given by the equalities $F_{p}^{(1)}\left(\delta q_{\perp}\right)=F_{p}^{(1)}(\delta \phi)$ and $F_{p}^{(2)}\left(\delta q_{\perp}, \delta y\right)=$ $F_{p}^{(2)}(\delta \phi, \delta y)$ in the limit $M \gg 1\left(\delta y \sim M^{-1}, \delta q_{\perp} \sim M^{-1}, \delta \phi \sim M^{-1}\right)$, (b) introducing the index $\eta=\frac{D-D_{F}}{D}$ as a measure of intermittency strength, eqs.(4.9) with $\delta>2$ show that the weakest effect appears in $1 D$ intermittency in rapidity $\left(\eta=1-D_{1}\right)$ and the strongest in $2 D$ intermittency in transverse momentum plane $\left(\eta=\frac{D_{2}}{2}\right)$, and (c) the observed universality of $3 D$ intermittency index at present experim [12] may be attributed to the universal character of $D_{1}, D_{2}$ and in Fialkowski's notation, one may write $\phi_{2}=\frac{1}{3}\left(1+D_{2}-D_{1}\right)$.

In summary, we have found, under a certain number of simplified assumptions, a complete multidimensional intermittency pattern dependent on two universal indices $D_{1}, D_{2}$, which, we claim, is the most general pattern associated to a second-order quark-hadron phase transition.

\section{Phenomenology}

At present experiments the energy density in hadron-hadron or nucleus-nucleus collisions is not high enough to generate events of hadronized quark-gluon plasma in abundance and therefore the universal pattern (4.9) has to be modified by adding non-universal terms within the context of a two-component model [13]. These terms represent conventional, non-singu correlations which contribute to the factorial moments as follows 13]:

$$
F_{p}^{(D)}(M)=\lambda_{c} c_{p}^{(D)} M^{\left(D-D_{F}\right)(p-1)}+G_{p}^{(D)}(M)
$$

where $G_{p}^{(D)}(M)$ are slowly varying functions of $M$, and $\lambda_{c} \ll 1$ is the probability for producing quark-gluon plasma in the actual experiment $\left(\lambda_{c}=\frac{\sigma_{q-g}}{\sigma_{i n}}\right)$. In a simplified version of this model one may assume that the conventional components $G_{p}^{(D)}(M)$ are constant ( $M$-independent) terms $G_{p}^{(D)}$ and thus obtain a generalized Fialkowski's pattern as follows [12]:

$$
\ln \left(F_{p}^{(D)}-G_{p}^{(D)}\right)=\left(\frac{D-D_{F}}{D}\right)(p-1) \ln \left(M^{D}\right)+\text { constant }
$$


valid for all moments $(p=2,3, \ldots)$ and all dimensions $(D=1,2,3)$. Hence the modified linear forms (5.2) are suitable for phenomenological comparison with present experiments in order to reveal the universal indices $\eta=\frac{D-D_{F}}{D}$ of the pattern (4.9). In the particular case of $3 D$ intermittency, Fialkowski has verified the universality of the index $\eta$ by comparing the linear form (5.2) for $p=2, D=3$ with measurements corresponding to a number of processes. In his notation $\left(\eta=\phi_{2}\right.$ for $p=2$ and $D=3)$ the universal index $\phi_{2}$ is fixed by the data in the range $\phi_{2}=0.4-0.5$ [12]. For phenomenological purposes we may now express the fractal dimensions $\left(D_{1}, D_{2}\right)$ in terms of the universal indices $\left(\phi_{2}, \delta\right)$, using the relations: $D_{1}+D_{2}=\frac{3 \delta}{1+\delta}, \quad \phi_{2}=$ $\frac{1}{3}\left(D_{2}-D_{1}+1\right)$. We find:

$$
D_{1}=-\frac{3 \phi_{2}}{2}+\frac{1}{2}+\frac{3 \delta}{2(1+\delta)} \quad D_{2}=\frac{3 \phi_{2}}{2}-\frac{1}{2}+\frac{3 \delta}{2(1+\delta)}
$$

Equations (5.3) together with the constraints $0<D_{1}<1,1<D_{2}<2, \delta>2$ put a restriction on the index $\phi_{2}$ :

$$
-\frac{1}{3}+\frac{\delta}{1+\delta}<\phi_{2}<\frac{5}{3}-\frac{\delta}{1+\delta}
$$

leading to a maximal range of values allowed for $\phi_{2}, \frac{1}{3}<\phi_{2}<1$. The lower bound gives a measure of $3 D$ intermittency strength and it is remarkable that the phenomenological values of $\phi_{2}$ lie in this domain, close to the lower bound [12. The bounds (5.4) on the other hand show a qualitative interrelation of the measurable index $\phi_{2}$ with the critical exponent $\delta$ and may lead to a phenomenological estimate of the allowed values for this fundamental parameter. In fact, for two distinct cases (a) $\delta=3$ corresponding to the mean-field approximation and (b) $\delta \approx 5$ corresponding to the universality class of the $n=4$ Heisenberg magnet $\left(M \sim H^{\frac{1}{\delta}}\right)$ suggested by Wilczek [2], we have (a) $\frac{5}{12}<\phi_{2}<\frac{11}{12}$ and (b) $\frac{1}{2}<\phi_{2}<\frac{5}{6}$ respectively. We observe that Fialkowski's phenomenological estimate of $\phi_{2}$ is consistent with the mean-field approximation value $\delta=3$ but marginally consistent with the $n=4$ magnet value $\delta \approx 5$. It seems that present data favours rather small values of the critical exponent $\delta \leq 4$, close to the mean-field approximation value $\delta=3$ [Fig.1]. For completeness we

Figure 1: The bounds eq.(5.4) in a $\delta$ - $\phi_{2}$ plot together with Fialkowski's values for $\phi_{2}$ (shaded region).

may write similar bounds for $1 D$ and $2 D$ intermittency indices, $\eta=\frac{D-D_{F}}{D}$. For this purpose and according to the pattern (4.9) we put $\eta_{1}=1-D_{1}(\delta y), \eta_{1 \perp}=D_{2}-1(\delta \phi$ or $\left.\delta q_{\perp}\right)$ for $1 D$ intermittency and $\eta_{2}=\frac{D_{2}-D_{1}}{2}\left(\delta y \delta q_{\perp}\right.$ or $\left.\delta y \delta \phi\right) \eta_{2 \perp}=\frac{D_{2}}{2}\left(\delta q_{\perp} \delta \phi\right)$ for $2 D$ intermittency. Following the same procedure as in the case of $3 D$ intermittency we find, for a given value of the critical exponent $\delta$, the bounds

$$
\begin{aligned}
& 1 D: 0<\eta_{1}<3-\frac{3 \delta}{1+\delta} ; \frac{3 \delta}{1+\delta}-2<\eta_{1 \perp}<1 \\
& 2 D: \frac{3 \delta}{2(1+\delta)}-1<\eta_{2}<2-\frac{3 \delta}{2(1+\delta)} ; \frac{3 \delta}{2(1+\delta)}-\frac{1}{2}<\eta_{2 \perp}<1
\end{aligned}
$$


showing a strong $2 D$ intermittency effect in the transverse momentum plane $\left(\frac{1}{2}<\right.$ $\left.\eta_{2 \perp}<1\right)$. Finally, the phenomenological capacity of our approach is illustrated in Fig. 2 considering as a basic ingredient Fialkowski's universality for $3 D$ intermittency at the level of the second factorial moment $F_{2}^{(3)}(M)$. In Fig. 2a the consistency of the bounds (5.4) with the trend of the data is shown and the accumulation tendency towards the lower bound $\phi_{2}=\frac{1}{3}$ is illustrated. We have used the outcome of a recent analysis of Fialkowski, based on NA35 measurements [14 related to a number of processes $(O A u, S S, S A u)$. In Fig. 2b a particular multidimensional intermittency pattern is singled-out corresponding to the choice $\delta=3$ (mean-field approximation) and $\phi_{2}=0.44$ (average value from Fialkowski's analysis) in the general solution (4.9). This illustration suggests that a phenomenological exploitation of our model would require an extension of Fialkowski's analysis taking into consideration measurements of $1 D$ and $2 D$ patterns as well. In such a treatment one could be able not only to reveal a universal multidimensional intermittency effect associated to a second-order quark-hadron phase transition, but also to measure the critical exponent $\delta$ since, for a given value of $\phi_{2}$, the slopes of $1 D$ and $2 D$ moments in Fig. $2 \mathrm{~b}$ are sensitive to the actual value of $\delta$.

Figure 2: (a) The moments (5.2) for $p=2, D=3$ corresponding to the limiting slopes $\phi_{2}=1 / 3,1$ and (b) a particular multidimensional intermittency pattern eq.(4.9) with $\delta=3, \phi_{2}=0.44$, are compared with Fialkowski's data analysis. The power laws are arbitrarily normalized at the same point.

\section{Conclusions}

In this work we have studied the general structure of multidimensional intermittency patterns in hadronic collisions generated by a second-order quark-hadron phase transition. For this purpose we have searched for the most general class of fractals in momentum space associated to self-similar structures in configuration space normally developed at $T=T_{c}$ [3]. The significance of self-similarity in configuration s pace (space-time) for intermittency phenomena has been recently emphasized by Bialas in connection with the Bose-Einstein interference effect [15]. A basic ingredient in our approach is the standard inside-outside cascade picture for the development of the collision, in the hadronization sector, allowing the use of rapidity as a longitudinal coordinate both in configuration and momentum space $(\xi \approx y)$. In this framework, employing the reduction formalism for the inclusive $S$-matrix, an integral representation connecting hadronic densities in momentum and configuration space was established. We have found that the general fractal solution both in momentum and configuration space has the geometrical structure of a Cartesian product of two fractals, one in rapidity space and the other in the transverse-momentum or impactparameter space respectively. The resulting multidimensional intermittency pattern 
has a linear spectrum of indices, as expected, dependent on two universal parameters $\left(D_{1}, D_{2}\right)$, geometrically identified with the fractal dimensions in rapidity $\left(D_{1}\right)$ and impact parameter space $\left(D_{2}\right)$. Physically these universal indices are constrained by the isothermal critical exponent $\delta$ of the strongly interacting system at $T=T_{c}$, $D_{1}+D_{2}=\frac{3 \delta}{1+\delta}$. For realistic values $\delta>2$ we have seen that $1 D$ projections of the fractal system in the transverse-momentum plane are also fractals with fractal dimension $\tilde{D}_{\perp 1}=2-D_{2}$. Hence $1 D$ intermittency in azimuthal angle $(\delta \phi)$ or transverse momentum $\left(\delta q_{\perp}\right)$ reflects the development of a $2 D$ fractal structure in transverse momentum plane with fractal dimension $\tilde{D}_{2}=\tilde{D}_{\perp 1}<1$. In this case $1 D$ intermittency (in $\delta \phi$ or $\left.\delta q_{\perp}\right)$ is weaker than the corresponding effect in $2 D\left(\delta \phi \delta q_{\perp}\right)$ in accordance with previous proposals about the dependence of intermittency strength on dimensionality in momentum space [16]. On the contrary, $1 D$ intermittency in rapidity is genuine effect since the fractal structure $\tilde{F}_{1}$ in the Cartesian product $\tilde{F}=\tilde{F}_{1} \times \tilde{F}_{2}$ eq.(3.17) is an intrinsic property of the critical system and not the artefact of a structure in higher dimensions. Its strength, in particular, depends on the basic index $D_{1}$ and is not generated by projecting out fractals embedded in higher dimensions.

Our approach to intermittency, based on the critical properties of the strongly interacting system, is suitable for hadronic collisions $(h h)$ and especially for relativistic heavy-ion processes $(A A)$ with high energy-density. It is complementary to perturbative QCD-inspired treatments, mainly applicable to $e^{+} e^{-}$intermittency where a branching mechanism at the parton level is expected to dominate the density fluctuations of the produced hadrons [17].

The phenomenological implications of our solution eq.(4.9) can only be revealed after subtracting the conventional contributions to factorial moments in the framework of a two-component model [13]. A simplified version of this treatment leads naturally to Fialkowski's universality in $3 D$; in order to derive a direct connection of measurable intermittency indices with the critical exponent $\delta$ at $T=T_{c}$ we have argued that an extension of Fialkowski's analysis in one and two dimensions is needed.

Finally, it is of interest to note that a natural extension of our approach from static to dynamic scaling hypothesis could easily accommodate non-trivial modifications of the intermittency pattern eq.(4.9), at least at the level of second moments $F_{2}^{(D)}$, due to the presence of the dynamic critical exponent [18]. It is tempting to associate such modifications with a more realistic picture of inside-outside hadronization process as a critical phenomenon out of equilibrium [18, 19]

In conclusion, we have shown that multidimensional intermittency phenomena in hadronic collisions may originate from a simple pattern of fractal structures (monofractals) in configuration space (space-time) characteristic of a second-order phase transition. A careful study of these phenomena is therefore necessary, both theoretically and experimentally, in an attempt to establish a link between criticality of the underlying theory (QCD at $T=T_{c}$ ) and complexity of the hadronic system at the transition temperature $\left(S\right.$-matrix at $\left.T=T_{c}\right)$.

\section{References}

[1] T. Hatsuda, Nucl. Phys. A544 (1992) 27. 
[2] F. Wilczek, Int. J. Mod. Phys. A7 (1992) 3911.

[3] R.B. Stinchcombe, Order and Chaos in Nonlinear Physical systems p.295, Edited by S. Lundquist, N.H. March and M.P. Tosi, Plenum Press, New York 1988.

[4] J.D. Bjorken, Phys. Rev. D27 (1983) 140;

N.G. Antoniou, F.K. Diakonos and I.S. Mistakidis, Phys. Lett. B293 (1992) 187.

[5] P. Carruthers and I. Sarcevic, Phys. Rev. Lett. 63 (1989) 1562.

[6] A.H. Mueller, Phys. Rev. D2 (1970) 2963.

[7] H. Satz, Nucl. Phys. B326 (1989) 613 ;

J. Wosiek, Acta Phys. Pol. B19 (1988) 863 ;

N.G. Antoniou, E.N. Argyres, A.P. Contogouris, C.G. Papadopoulos and S.D.P. Vlassopulos, Phys. Lett. B245 (1990) 619 ;

N.G. Antoniou, A.P. Contogouris, C.G. Papadopoulos and S.D.P. Vlassopulos, Phys. Rev. D45 (1992) 4034 ;

A. Bialas and R.C. Hwa, Phys. Lett. B253 (1991) 436.

[8] K. Falconer, Fractal Geometry, Wiley, New York 1989

[9] H.E. Stanley, Phase Transitions and Critical Phenomena Clarendon Press, Oxford 1971 ;

S-K. Ma, Modern Theory of Critical Phenomena Benjamin Publishing Co., Reading /Mass. 1976 ;

M.I. Polikarpov, Phys. Lett. B236 (1990) 61.

[10] A. Bialas and R. Peschanski, Nucl. Phys. B273 (1986) 703; B308 (1988) 803.

[11] I.M. Dremin and M.T. Nazirov, preprint LUTP 92-27, 1992, to be published in Z. Phys. C.

[12] K. Fialkowski, Phys. Lett. B272 (1991) 139; Proc. Cracow Workshop on Multiparticle Production 1993, World Scientific, Singapore, to be published.

[13] N.G. Antoniou, E.N. Argyres, C.G. Papadopoulos and S.D.P. Vlassopulos, Phys. Lett. B260 (1991) 199.

[14] J. Bächler et al. ; NA35 Collaboration, Z. Phys. C57 (1993) 541.

[15] A. Bialas, Acta Phys. Pol. B23 (1992) 561.

[16] W. Ochs, Phys. Lett. B247 (1990) 101; Z. Phys. C 50 (1991) 339; A. Bialas and J. Seixas, Phys. Lett. B250 (1990) 191.

[17] W. Ochs and J. Wosiek, Phys. Lett. B289 (1992) 159 ;

Ph. Brax, J.L. Meunier and R. Peschanski, "Angular intermittency in QCD jets", Nice preprint INLN 93.01 (1993) and Saclay preprint SPth/93-011;

Yu.L. Dokshitzer and I.M. Dremin, preprint LU-TP 92-30, 1992, to be published in Nucl. Phys. B. 
[18] N.G. Antoniou, F.K. Diakonos, I.S. Mistakidis and C.G. Papadopoulos, in preparation.

[19] P. Carruthers, Summary talk presented at LESIP I, Local Equilibrium in Strong Interaction Physics, Bad Honnef, Germany, September 2-6, 1984;

K. Rajagopal and F. Wilczek, 'Quark Matter '93', Borlänge, Sweden, June 20-24, 1993. 